\title{
tsRNA-5001a promotes proliferation of lung adenocarcinoma cells and is associated with postoperative recurrence in lung adenocarcinoma patients
}

\author{
Fang $\mathrm{Hu}^{1 \#}$, Yanjie Niu ${ }^{1 \#}$, Xiaowei Mao ${ }^{1}$, Jiangtao Cui ${ }^{2}$, Xiaoting $\mathrm{Wu}^{3}$, Charles B. Simone $\mathrm{II}^{4}$, \\ Hye Seon Kang ${ }^{5}$, Wenxin Qin ${ }^{6}$, Liyan Jiang ${ }^{1}$
}

${ }^{1}$ Department of Pulmonary, Shanghai Chest Hospital, Shanghai Jiao Tong University, Shanghai, China; ${ }^{2}$ Department of Thoracic Surgery, Shanghai Chest Hospital, Shanghai Jiao Tong University, Shanghai, China; ${ }^{3}$ Department of Integrated Traditional Chinese and Western Medicine, Shanghai Chest Hospital, Shanghai Jiao Tong University, Shanghai, China; ${ }^{4}$ Department of Radiation Oncology, Memorial Sloan Kettering Cancer Center and New York Proton Center, New York, NY, USA; ${ }^{5}$ Division of Pulmonary, Critical Care and Allergy, Department of Internal Medicine, Bucheon St. Mary's Hospital, College of Medicine, The Catholic University of Korea, Seoul, Republic of Korea; ${ }^{6}$ State Key Laboratory of Oncogenes and Related Genes, Shanghai Cancer Institute, Renji Hospital, Shanghai Jiao Tong University School of Biomedical Engineering, Shanghai, China Contributions: (I) Conception and design: L Jiang, W Qin, F Hu, X Mao; (II) Administrative support: L Jiang, W Qin; (III) Provision of study materials or patients: L Jiang, Y Niu, F Hu; (IV) Collection and assembly of data: F Hu, Y Niu, X Mao; (V) Data analysis and interpretation: F Hu, X Mao, J Cui, X Wu; (VI) Manuscript writing: All authors; (VII) Final approval of manuscript: All authors.

\#These authors contributed equally to this work.

Correspondence to: Liyan Jiang. Department of Pulmonary, Shanghai Chest Hospital, Shanghai Jiao Tong University, 241 Huaihai West Road, Xuhui District, Shanghai 200030, China. Email: jiang_liyan2000@126.com; Wenxin Qin. State Key Laboratory of Oncogenes and Related Genes, Shanghai Cancer Institute, Renji Hospital, Shanghai Jiao Tong University School of Biomedical Engineering, Shanghai 200032, China; Shanghai Cancer Institute, Renji Hospital, Shanghai Jiao Tong University School of Medicine, Shanghai 200032, China. Email: wxqin@sjtu.edu.cn.

Background: The biological role and clinical significance of transfer RNA-derived small RNAs (tsRNAs) remain largely unclear. The purpose of this study was to investigate the biological function, molecular mechanism, and clinical significance of tsRNA-5001a in lung adenocarcinoma.

Methods: The function of tsRNA-5001a on the growth of tumor cells was accessed by cell function experiments. Real-time quantitative polymerase chain reaction (RT-qPCR) was used to detect the expression of tsRNA-5001a in paired samples of lung adenocarcinoma. Cell localization of tsRNA-5001a was performed by nuclear-cytoplasmic separation assay. Transcriptome sequencing was used to screen the molecules involved in the regulatory network of tsRNA-5001a. Independent samples $t$-test was used to compare the two groups. Prism software (Prism 7.0) was used to analyze the statistical results. $\mathrm{P}<0.05$ was considered statistically significant.

Results: tsRNA-5001a was significantly upregulated in lung adenocarcinoma tissues. Upregulation of tsRNA-5001a was found to increase the risk of postoperative recurrences in patients with lung adenocarcinoma and was associated with poor prognosis. Function assay showed that overexpression tsRNA5001a could significantly promote cell proliferation. In contrast, knockdown of tsRNA-5001a significantly inhibited the proliferation of lung adenocarcinoma cells. In addition, nucleoplasmic isolation assay indicated that tsRNA-5001a was located mainly in the cytoplasm. According to the results of RNA sequencing and The Cancer Genome Atlas database (TCGA database) analysis, growth arrest and DNA damage 45G (GADD45G) was screened and may be the target gene of tsRNA-5001a.

Conclusions: tsRNA-5001a promotes the proliferation of lung adenocarcinoma cells and increases the risk of postoperative recurrences in lung adenocarcinoma patients.

Keywords: tRNA-derived fragments; tsRNA-5001a; lung adenocarcinoma; proliferation; recurrence

$\wedge$ ORCID: 0000-0003-2586-9266. 
Submitted Jul 29, 2021. Accepted for publication Oct 22, 2021.

doi: $10.21037 /$ tlcr-21-829

View this article at: https://dx.doi.org/10.21037/tlcr-21-829

\section{Introduction}

Over the past half century, the worldwide incidence and mortality rate of lung cancer have sharply increased $(1,2)$. Lung adenocarcinoma is the most frequently occurring histological subtype of malignancy of the lung, accounting for approximately $40-50 \%$ of lung cancer cases (3).

The occurrence and development of lung adenocarcinoma are closely related to the abnormal regulation of gene expression. Past studies investigating lung adenocarcinoma have typically focused on protein-coding genes. With the development of high-throughput sequencing technology, the transcripts of noncoding proteins in the human transcriptome have been further established as contributors to the occurrence and development of many diseases. Studies have demonstrated that about $90 \%$ of the genes in the human genome are transcribed, but only $1-3 \%$ of the transcription products have protein-encoding functions. Those RNAs without protein-encoding functions are called non-coding RNAs (ncRNA) (4). ncRNAs can perform various special regulatory functions at the RNA level (5). Among them, transfer RNA-derived small RNAs (tsRNAs) are newly discovered small RNA fragments with biological functions derived from tRNA. tsRNA is a small RNA fragment of a specific size, which is produced by cutting the loop of tRNA by specific nucleases [such as Dicer and angiopoietin (ANG)] in cells and tissues or under specific conditions (6). Recent studies have shown that tsRNAs can regulate gene expression at transcriptional and posttranscriptional modifications and epigenetic inheritance levels (7).

After the discovery of tsRNA, some studies have shown that its abnormal expression is associated with a variety of human disease processes, implying that tsRNA can play a role in several human diseases (8-11). Of particular concern is the evidence that some specific tsRNAs are abnormally expressed in a variety of tumors, such as breast cancer, hematological malignancies, lung cancer, and prostate cancer $(7,9,10,12)$. Although research on ncRNA has made rapid progress in recent years, the relationship between tsRNAs and tumorigenesis is still unclear, particularly in regard to the role and mechanism relevant to the occurrence, development, invasion, and metastasis of lung adenocarcinoma. A previous study has detected the differential expression level of tsRNA in lung adenocarcinoma and adjacent tissues by using nextseq system. The results showed that 34 tsRNA subtypes were abnormally expressed in lung adenocarcinoma. Further study found that the abnormal expression of tsRNA may be closely related to the pathogenesis and development of lung adenocarcinoma (13). In our previous study, we used Arraystar Human tRF\&tiRNA PCR Array technology to analyze and isolate a set of tsRNAs related to lung adenocarcinoma. Cell function experiments were able to identify tsRNA-5001a as exerting several biological functions. Therefore, the purpose of this study was to explore the impact of tsRNA-5001a on the occurrence and development of lung adenocarcinoma and to investigate the clinical value of tsRNA-5001a in judging the prognosis of lung adenocarcinoma. The findings of this study may provide novel sources for potential lung adenocarcinoma diagnostic markers and therapeutic targets.

tsRNA is a new and relatively blank research field. As a molecule in an emerging field, tsRNA-5001a has not been studied before. Compared with previous studies, the molecule studied in this study was relatively novel. Moreover, this study explored the function of tsRNA5001a in lung adenocarcinoma at the cellular, molecular and clinical levels, and predicted the possible target genes of tsRNA-5001a combined with RNA-seq detection data and TCGA database, which has a certain potential clinical application value. We present the following article in accordance with the MDAR reporting checklist (available at https://dx.doi.org/10.21037/tlcr-21-829).

\section{Methods}

\section{Patients and clinical assessments}

Patients with lung adenocarcinoma who underwent lung cancer resection in Shanghai Chest hospital in 2016 and who harbored epidermal growth factor receptor (EGFR)sensitive mutations (19 deletion or 21L858R mutations) 
were enrolled. The enrolled patients were staged according to the eighth edition of the tumor-node-metastasis (TNM) staging system.

All patients underwent a systemic examination for assessment of their general condition before surgery. Chest computed tomography (CT), abdominal B-ultrasound, hematology examination, and for more advanced clinical stages, brain magnetic resonance imaging (MRI), bone scan, or positron emission tomography-computed tomography (PET-CT) were conducted for postoperative follow-up examination. Clinical characteristics, including age, gender, smoking status, EGFR mutation type, TNM stage, and the pathological features of solid-type and micropapillary, were collected. Disease-free survival (DFS) was defined as the interval between receiving surgery and the date of recurrence or last follow-up visit. Overall survival (OS) was defined as the interval between receiving surgery and death from any cause or last follow-up visit.

\section{$R N A$ extraction and pretreatment of tissue samples}

Overall, 44 primary lung adenocarcinoma tumor tissues and paired paracancerous tissues were collected from patients who had received surgery at Shanghai Chest Hospital. Samples were collected from January 2016 to December 2016 with the consent of patients and approval from the Ethics Committee of the Shanghai Chest Hospital, Shanghai Jiao Tong University (No. KS(Y)21105). All procedures performed in this study involving human participants were in accordance with the Declaration of Helsinki (as revised in 2013). All paired samples of cancer tissues and paracancerous tissues were confirmed by 2 pathologists independently and stored at $-80{ }^{\circ} \mathrm{C}$.

Total RNAs from tissue samples were extracted using RNAiso Plus (cat. no. 9108; Takara; Shiga, Japan). A sterile surgical blade was used to cut a tissue block about $5 \mathrm{~mm}$ thick, which was place in a $1.5-\mathrm{mL}$ Eppendorf (EP) tube. After $1,000 \mu \mathrm{L}$ of RNAiso Plus was added and beads were ground, the tissue block was placed on the grinding instrument and ground for 15 minutes according to the instructions of the instrument. The lysate was extracted with $200 \mu \mathrm{L}$ trichloromethane and centrifuged at $12,000 \mathrm{rpm}$ and $4{ }^{\circ} \mathrm{C}$ for 10-15 minutes. The supernatant was then transferred into a new $1.5 \mathrm{~mL}$ EP tube with a pipette gun, and $1,000 \mu \mathrm{L}$ of isopropanol was added, then vortexed to mix thoroughly, and then centrifuged at $12,000 \mathrm{rpm}$ at $4^{\circ} \mathrm{C}$ for $10-15$ minutes. The supernatant was discarded after centrifugation. The precipitate was then washed twice with $75 \%$ alcohol. After drying, the RNA was dissolved with diethyl pyrocarbonatetreated water (DEPC water, RNase-free water) and then stored at $-80^{\circ} \mathrm{C}$

The rtStarTM tRF\&tiRNA Pretreatment Kit (Cat no. AS-FS-005; ArrayStar, Rockville, MD, USA) was used to remove tsRNA modifications that interfere with the small RNA complement DNA (cDNA) library construction of real-time quantitative PCR (RT-qPCR) according to the manufacturer's instructions. This pretreatment kit removes 3'-aminoacyl (charged) and 3'-cP to 3'-OH for 3' adaptor ligation; phosphorylates 5'-OH (hydroxyl group) to $5^{\prime}-\mathrm{P}$ for 5 '-adaptor ligation; and demethylates $\mathrm{m} 1 \mathrm{~A}, \mathrm{~m} 1 \mathrm{G}$, and $\mathrm{m} 3 \mathrm{C}$ for efficient reverse transcription.

\section{Reverse transcription and quantitative real-time PCR}

Total RNAs were subjected to cDNA synthesis by Mir-X miRNA First-Strand Synthesis (cat. no. 638315, Takara; Shiga, Japan) according to the manufacturer's protocol. qPCR was performed with SYBR Green Master (cat. no. 4913914001, Roche; Basel, Switzerland) using an AB ViiA 7 real-time PCR instrument (Applied Biosystems, Thermo Fisher Scientific; Waltham, Massachusetts, USA). U6 was chosen as internal control for tsRNA quantification in tissue samples. The forward primers for tsRNA-5001a were obtained from RiboBio Company (Guangzhou, China). The mRQ 3' Primer was used as reverse primer in tsRNA-5001a qPCR. The U6 forward and reverse primers and mRQ 3' primer were provided by the Mir-X miRNA First-Strand Synthesis kit (cat. no. 638315, Takara; Shiga, Japan). The relative expression levels of tsRNA-5001a were calculated by the $2^{-\Delta \Delta \mathrm{Ct}}$ method. The thermocycling conditions were as follows: $95^{\circ} \mathrm{C}$ for 5 minutes for 1 cycle and $95{ }^{\circ} \mathrm{C}$ for 10 seconds and $60^{\circ} \mathrm{C}$ for 30 seconds for 40 cycles. This was followed by the dissolution curve stage, which is a default setting of the instrument (1 cycle) (Applied Biosystems, Thermo Fisher Scientific; Waltham, Massachusetts, USA).

\section{Cell culture}

A549 lung adenocarcinoma cells were purchased from the Shanghai cell bank of the Chinese Academy of Sciences (cat. no. TCHu150; Shanghai, China), and PC9 lung adenocarcinoma cells were purchased from FuHeng Cell Center (cat. no. FH00831; Shanghai, China). A549 and PC9 cells were cultured in modified HyClone RPMI 1640 medium (cat. no. SH30809.01; HyClone, Logan, Utah, USA) supplemented with $10 \%$ fetal bovine serum 
(FBS; cat. no. 04-001-1ACS; Biological Industries USA, Cromwell, CT, USA) and 1\% penicillin-streptomycin (cat no. SV30010; HyClone, Logan, Utah, USA) at $37^{\circ} \mathrm{C}$ in a $5 \%$ $\mathrm{CO}_{2}$ incubator. All cell-related experiments were performed in a dedicated cell room of the Central Laboratory of Shanghai Chest Hospital.

\section{Overexpression and knockdown of tsRNA-5001a by transfection with mimic and inbibitor}

Overexpression and knockdown of tsRNA-5001a were accomplished by transfecting tsRNA-5001a mimics and antagomirs (modified inhibitors) into cells, respectively.

The specific steps of transfection were as follows: $400 \mu \mathrm{L}$ of transfection buffer was added into an EP tube, $22 \mu \mathrm{L}$ $(20 \mu M)$ of tsRNA-5001a mimic or antagomir or their blank control was added and vortex for 10 seconds until fully mixed, and $8 \mu \mathrm{L}$ of transfection reagent was added and mixed well. Subsequently, the mixture was left to stand for 10 minutes at room temperature. The mixture was then added into the prepared cells to be transfected (generally, the cell density was controlled at about 50-60\%). The transfection buffer and transfection reagent were provided by the transfection kit (ref no. PT-114-15, jetPRIME, Polyplus Transfection; Illkirch, France). tsRNA-5001a mimic and the corresponding negative control (NC) sequence (Sangon Biotech, Shanghai, China) were as follows: tsRNA-5001a: 5'-CCUUCGAUAGCUCAG-3'; NC: 5'-GACUUAAGCGGCUACAUA-3'.

The chemically modified antagomir complement to tsRNA-5001a was designed to inhibit endogenous tsRNA5001a expression, and the antagomir NC was obtained from RiboBio Company. The sequences for antagomir and antagomir NC (were as follows: antagomir-tsRNA5001a: 5'-CUGAGCUAUCGAAGG-3'; antagomir NC: 5'-CAGUACUUUUGUGUAGUACAAA-3'.

The expression of growth arrest and DNA damage 45G $(G A D D 45 G)$ in overexpressed and knockdown cell lines was detected by RT-qPCR. The forward primer sequence of GADD45G was 5'-CTAGCTGCTGGTTGATCGCA-3', and the reverse primer sequence of $G A D D 45 G$ was 5'-TTTGGCTGACTCGTAGACGC-3'.

\section{Cell behavior assays}

For the cell proliferation assay, $2 \times 10^{3}$ cells were seeded on $96-$ well plates and cultured for 1-5 days. Each well was incubated with $10 \mu \mathrm{L}$ cell counting kit-8 (CCK-8) detection solution and
$90 \mu \mathrm{L}$ serum-free medium for 1 hour and 15 minutes (cat no. BS350B Biosharp; Beijing, China). The absorbance values at $450 \mathrm{~nm}$ were then measured. In the colony formation assay, 500 cells were seeded in $2 \mathrm{~mL}$ of complete culture media in a 6-well plate for 10-14 days (the proliferation rate of PC9 cells is slower than that of A549 cells, and the culture time is relatively longer). After 10-14 days, colonies were stained using crystal violet and counted after drying.

\section{tsRNA-5001a localization}

Cell localization of tsRNA-5001a was performed by nuclearcytoplasmic separation assay. Nuclear and cytoplasmic RNA were isolated using PARIS ${ }^{\mathrm{T}}$ kit according to the manufacture's instruction (AM1921; Invitrogen, Thermo Fisher Scientific, USA). The expression of tsRNA-5001a in the nucleus and cytoplasm was detected by RT-qPCR. U1 and $G A P D H$ was used as nuclear and cytoplasmic references, respectively, to detect whether the nucleocytoplasmic separation was complete. The forward primer sequence of U1 was 5'-GGGAGATACCATGATCACGAAGGT-3', and the reverse primer sequence of $U 1$ was 5'-CCACAAATTAT GCAGTCGAGTTCCC-3'. The forward primer sequence of GAPDH was 5'-AGATCATCAGCAATGCCTCCTG-3', and the reverse primer sequence of $G A P D H$ was 5'-GCAGGGATGATGTTCTGGAGAG-3'.

\section{$R N A$ sequencing}

The Illumina Xten platform (San Diego, California, USA) was used for sequencing. After quality control was performed, the original sequencing data were mapped to the reference genome sequence, and the reads that were mapped to the gene were counted. After the gene expression level and gene expression differences were calculated, functional enrichment analysis of Gene Ontology (GO), clusters of euKaryotic Orthologous Groups (KOG), and Kyoto Encyclopedia of Genes and Genomes (KEGG) pathways were analyzed. The RNA sequencing (RNA-seq) samples used in this study included the knockdown group and its control, with three samples repeated in each group.

\section{Statistical analysis}

The data are expressed as mean \pm standard deviation. Each experiment was repeated at least 3 times. Independent samples $t$-test was used to compare the two groups. Clinical information was analyzed using $\chi^{2}$ tests. The correlation of the expression of 
Table 1 Clinical characteristics of the patients

\begin{tabular}{|c|c|c|c|c|}
\hline Characteristic & \multicolumn{3}{|c|}{ Patients $(n=44)$} & Total \\
\hline Median age [range] & 57 [37-68] & 63 [44-77] & 0.409 & $44[37-77]$ \\
\hline Gender, n (\%) & & & 0.322 & \\
\hline Male & $5(22.7)$ & $8(36.4)$ & & $13(29.5)$ \\
\hline Smoking status, n (\%) & & & 0.635 & \\
\hline Smoker & $2(9.1)$ & $3(13.6)$ & & $5(11.4)$ \\
\hline Never-smoker & $20(90.9)$ & $19(86.4)$ & & $39(88.6)$ \\
\hline Solid type, n (\%) & & & 0.540 & \\
\hline Micropapillary, n (\%) & & & 0.540 & \\
\hline Yes & $14(63.6)$ & $12(54.5)$ & & $26(59.1)$ \\
\hline No & $8(36.4)$ & $10(45.5)$ & & $18(40.9)$ \\
\hline EGFR mutation, $\mathrm{n}(\%)$ & & & 0.128 & \\
\hline 19del & $12(54.5)$ & 7 (31.8) & & $19(43.2)$ \\
\hline 21L858R & $10(45.5)$ & $15(68.2)$ & & $25(56.8)$ \\
\hline TNM stage, n (\%) & & & 0.082 & \\
\hline$|A-I| A$ & $3(13.6)$ & $8(36.4)$ & & $11(25.0)$ \\
\hline
\end{tabular}

${ }^{a}$, taking the median of tsRNA-5001a expression in tissues of all enrolled patients as the cutoff value, patients were divided into a high expression group and a low expression group. EGFR, epidermal growth factor receptor; TNM stage, tumor-node-metastasis stage.

tsRNA-5001a with clinicopathological features was calculated by Pearson's correlation test. The Kaplan-Meier method was used for univariate analysis. Prism software (Prism 7.0; San Diego, California, USA) was used to analyze the statistical results. A $\mathrm{P}$ value $<0.05$ was considered statistically significant.

\section{Results}

Arraystar Human tRF\&tiRNA PCR Array technology was used to analyze 3 pairs of cancer and paracancerous surgical samples of lung adenocarcinoma patients, and isolated a set of tsRNAs related to lung adenocarcinoma, the microarray data from which are owned by Yu Chengtao. With his consent, we further screened the microarray data and obtained tsRNA-5001a for further study. Since his research related to the chip has not been published, the detailed data of the chip are not shown in this study. Using the multiples of differences in $\mathrm{P}$ value $<0.05$ and $\mid \log 2 \mathrm{FCl}>1$ as the screening conditions, we screened out 40 significantly differentially expressed tsRNAs. For the selected upregulated tsRNA molecules in lung adenocarcinoma tissues, we used the cell function test to further clarify the biological function of tsRNA-5001a.

\section{Expression of tsRNA-5001a in tissue samples}

To evaluate the expression of tsRNA-5001a in lung adenocarcinoma cells, RT-qPCR assays were performed in 44 patients with lung adenocarcinoma who had matched paracancerous tissues. The clinical characteristics of the patients are presented in Table 1 . The $\chi^{2}$ test showed that clinical data. including age, gender, smoking status, solid- 


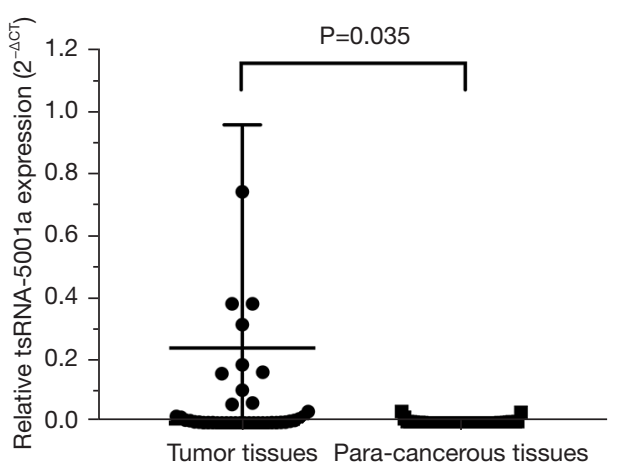

Figure 1 Expression of tsRNA-5001a in tumor tissues and paired paracancerous tissues was assessed by RT-qPCR. Symbols represent individual samples. Unpaired nonparametric $t$-test was used to evaluate significance. $\mathrm{P}<0.05$ was considered statistically significant. RT-qPCR, real-time quantitative polymerase chain reaction.

Table 2 Correlation of tsRNA-5001a expression with clinicopathological characteristics determined by Pearson's correlation analysis

\begin{tabular}{lcc}
\hline \multirow{2}{*}{ Parameters } & \multicolumn{2}{c}{ tsRNA-5001a expression } \\
\cline { 2 - 3 } & $\mathrm{R}$ & P value \\
\hline DFS & -0.432 & $0.003^{\mathrm{a}}$ \\
OS & -0.340 & 0.024 \\
Gender (male/female) & 0.296 & 0.051 \\
Age & -0.298 & 0.049 \\
TNM stage (IA-IIA/IIB and above) & 0.325 & 0.031 \\
Solid type (yes/no) & -0.147 & 0.34 \\
Micropapillary (yes/no) & -0.058 & 0.707 \\
Smoking status (yes/no) & 0.165 & 0.284 \\
EGFR mutation (19del/21L858R) & -0.271 & 0.075 \\
\hline
\end{tabular}

a, $\mathrm{P}<0.05$ was considered statistically significant. DFS, diseasefree survival; OS, overall survival; EGFR, epidermal growth factor receptor; TNM stage, tumor-node-metastasis stage.

type and micropapillary component, EGFR mutation type, and TNM stage, were well balanced among the high expression group and low expression groups $(\mathrm{P}>0.05)$.

The relative expression levels of tsRNA-5001a were detected using RT-qPCR, with the U6 housekeeping gene used as a control. The expression level of tsRNA-5001a in each tissue was calculated using the $2^{-\Delta \Delta C \mathrm{C}}$ method. Significant differences of tsRNA-5001a expression levels between tumor and paracancerous tissue were identified using the unpaired nonparametric $t$-test. The results revealed that the relative levels of tsRNA-5001a were significantly higher in the tumor tissue than in the paracancerous tissue $(\mathrm{P}=0.035$; Figure 1$)$. The expression level of tsRNA-5001a in tumor tissues relative to its respective paired paracancerous tissues of each enrolled patient is shown in Figure S1.

The correlation of the expression of tsRNA-5001a with clinicopathological features of patients with lung adenocarcinoma was calculated by Pearson's correlation test. As demonstrated in Table 2, the expression of tsRNA5001a was significantly correlated with DFS $(\mathrm{R}=-0.432$; $\mathrm{P}=0.003$; Figure $\mathrm{S} 2)$, $\mathrm{OS}(\mathrm{R}=-0.340 ; \mathrm{P}=0.024)$, age $(\mathrm{R}=-$ $0.298 ; \mathrm{P}=0.049)$, and TNM stage $(\mathrm{R}=0.325 ; \mathrm{P}=0.031)$.

The results of a Kaplan-Meier analysis for DFS and OS are shown in Figure 2. Survival analysis showed that the DFS time of the cohort with low tsRNA-5001a expression was significantly longer than that of the cohort with high tsRNA-5001a expression (Figure $2 A ; \mathrm{P}=0.002$ ). In addition, survival analysis curve showed that there was a numerical difference in the OS of the tsRNA-5001a low expression group and the high expression group, although this was not statistically significant (Figure 2B; $\mathrm{P}=0.234$ ).

\section{Overexpression of tsRNA-5001a promoted lung adenocarcinoma proliferation}

We used the jetPRIME kit to transfect the tsRNA-5001a mimic into A549 and PC9 lung adenocarcinoma cell lines to construct an overexpression transient cell line. After 24-48 hours of reaction, the overexpression efficiency was confirmed by RT-qPCR. The content of tsRNA-5001a in the overexpression group after transfection of A549 cells increased by 178 folds compared with that in the blank group, and the content of tsRNA-5001a in the overexpression group after transfection of PC9 cells increased by 32 folds compared with that in the blank group.

We used plate clone formation experiment and CCK-8 assay to detect cell proliferation. The plate clone formation experiment showed that tsRNA-5001a overexpression significantly promoted the proliferation of A549 cells (Figure 3A; $\mathrm{P}=0.001$ ) and $\mathrm{PC} 9$ cells (Figure 3B; $\mathrm{P}=0.002$ ). Moreover, the results of CCK-8 proliferation experiments also showed that overexpression of tsRNA-5001a could significantly promote the proliferation of A549 (Figure 3C; $\mathrm{P}=0.002$ ) and $\mathrm{PC} 9$ cells (Figure $3 D ; \mathrm{P}=0.001$ ). The above cell function experiments were mutually verified, and it was shown that the overexpression of tsRNA-5001a could promote the proliferation of lung adenocarcinoma cells. 

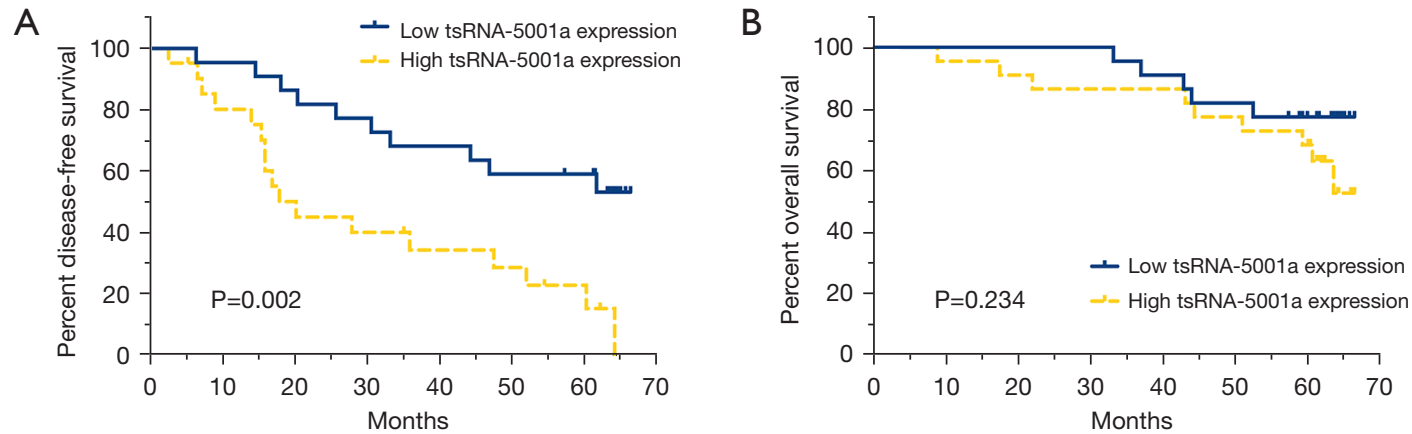

Figure 2 Kaplan-Meier curve analysis of tsRNA-5001a expression for DFS (A) and OS (B) in lung adenocarcinoma patients. Horizontal axis: DFS time (A) and OS time (B) in months. Vertical axis: DFS rate (A) and overall OS (B) in percentages. DFS, disease-free survival; OS, overall survival.

A

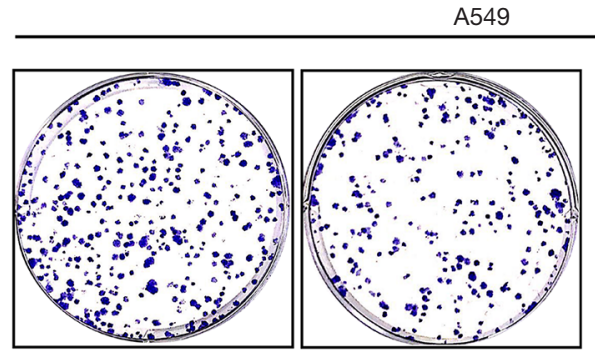

tsRNA-5001a overexpression

B

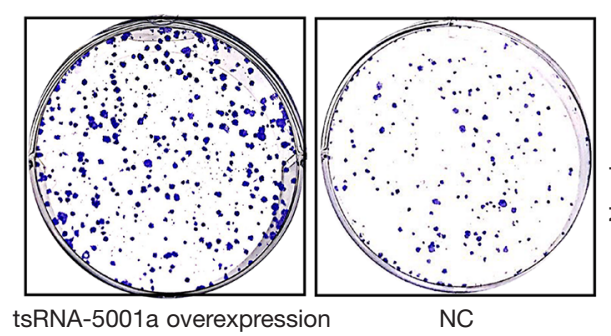

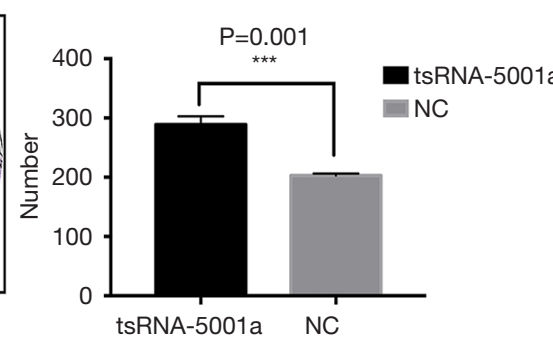

tsRNA-5001a NC

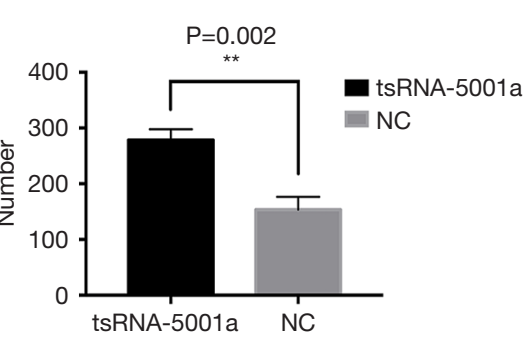

C

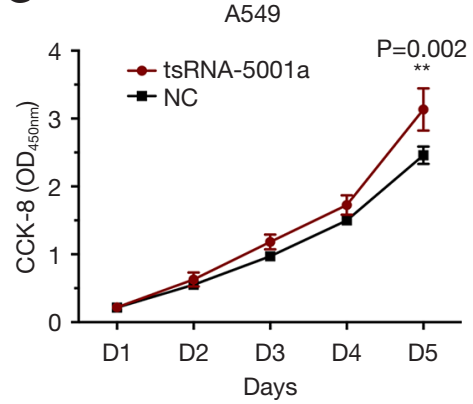

D

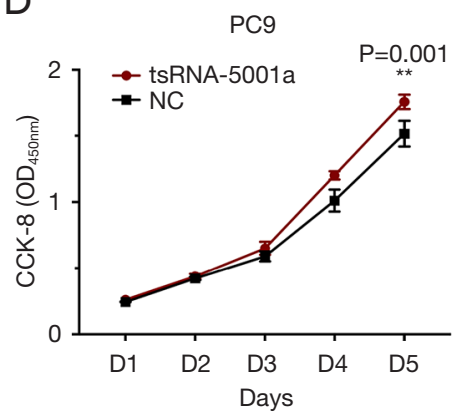

Figure 3 The results of functional test after overexpression of tsRNA-5001a in A549 and PC9 cell lines. After overexpression of tsRNA5001a in A549 cell line, the clone formation experiment showed that the cell clone formation ability was significantly increased (A), whereas the CCK-8 assay showed that the cell proliferation ability was significantly enhanced (C). Similarly, tsRNA-5001a overexpression in the PC9 cell line resulted in a significant increase in cell clone formation ability (B) and cell proliferation ability (D). Image J software was used to count the number of clones formed $(0.1 \%$ crystal violet staining; use six-well plates with 35 -mm diameter wells for colony formation experiments, magnification, $\times 1$ ). Absorbance at $450 \mathrm{~nm}$ was measured using a BioTek microplate reader. ${ }^{* *}, \mathrm{P}<0.01 ;{ }^{* * *}, \mathrm{P}<0.001$. CCK- 8 , cell counting kit-8; NC, negative control.

\section{Knockdown of tsRNA-5001a suppressed lung adenocarcinoma proliferation}

Subsequently, tsRNA-5001a antagomir or antagomir NC were transfected into A549 and PC9 lung adenocarcinoma cell lines to construct tsRNA-5001a knockdown cell lines. Cell proliferation was measured by CCK- 8 assay and plate clone formation experiments. The results of cell clone formation assay showed that knocking down tsRNA-5001a could reduce the A549 (Figure 4A; $\mathrm{P}=0.001$ ) and PC9 (Figure 4B; 
A

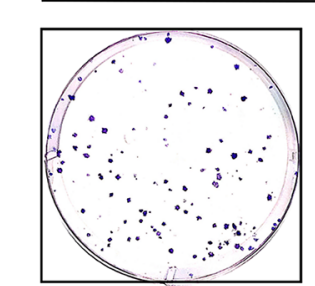

tsRNA-5001a knockdown

B

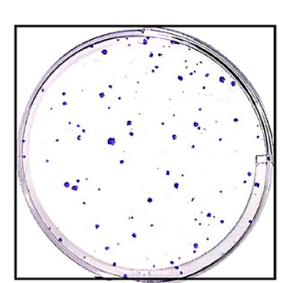

tsRNA-5001a knockdown
A549

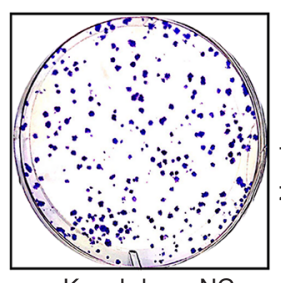

Knockdown NC

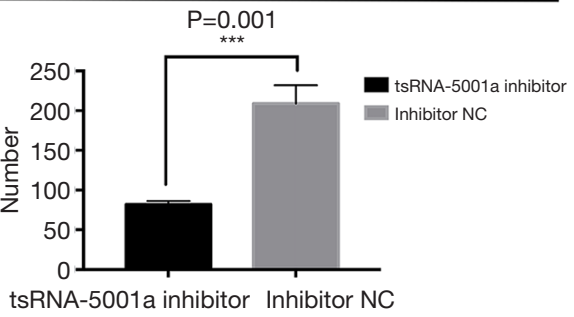

PC9

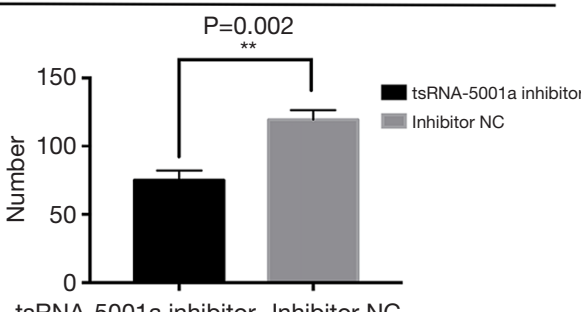

C

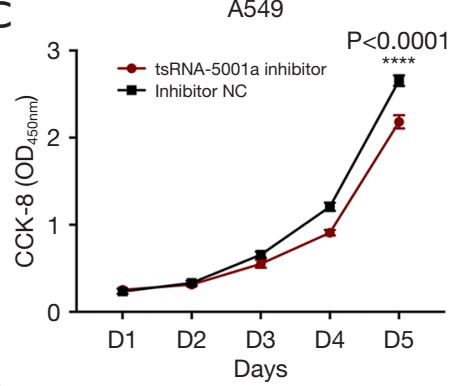

$\mathrm{D}$

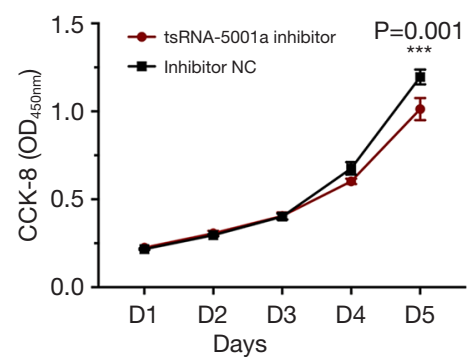

Figure 4 Functional test results after knocking down tsRNA-5001a in A549 and PC9 cell lines. After knocking down tsRNA-5001a in the A549 cell line, clone formation experiments showed that the cell clone formation ability was significantly reduced (A), while CCK-8 experiments showed that the cell proliferation ability was significantly weakened (C). Similarly, after knocking down tsRNA-5001a in the PC9 cell line, clone formation experiments showed that the cell clone formation ability was significantly reduced (B), whereas the CCK8 experiment showed that the cell proliferation ability was significantly weakened (D). Image $\mathbf{J}$ software was used to count the number of clones formed $(0.1 \%$ crystal violet staining; use six-well plates with $35-\mathrm{mm}$ diameter wells for colony formation experiments, magnification, $\times 1)$. Absorbance at $450 \mathrm{~nm}$ was measured using a BioTek microplate reader. ${ }^{* *}, \mathrm{P}<0.01$; ${ }^{* * *}, \mathrm{P}<0.001$, ${ }^{* * * *}, \mathrm{P}<0.0001$. CCK-8, cell counting kit-8; NC, negative control.

$\mathrm{P}=0.002$ ) cells clone formation ability. Furthermore, CCK-8 assay showed that after tsRNA-5001a was knocked down, the proliferation ability of both A549 cells (Figure 4C; $\mathrm{P}<0.0001$ ) and PC9 cells (Figure 4D; $\mathrm{P}=0.001$ ) decreased. Therefore, the results of CCK-8 assay further demonstrated that tsRNA5001a knockdown inhibited cell proliferation.

\section{tsRNA-5001a localization}

The nuclear and cytoplasmic RNA of A549 and PC9 cells were isolated and extracted by nucleo-cytoplasmic separation kits. The expression of U1 in nuclear RNA was significantly higher than that of $G A P D H$, whereas the expression of GAPDH in cytoplasmic RNA was significantly higher than that of $\mathrm{U} 1$, suggesting successful nucleocytoplasmic separation of RNA. The expression ratio of tsRNA-5001a in cytoplasm of A549 cells was $67.1 \%$ and that in nucleus was $32.9 \%$. In PC9 cells, the expression ratio of tsRNA-5001a was $82.1 \%$ in the cytoplasm and $17.9 \%$ in the nucleus. Nuclear and cytoplasmic fractionation examination revealed that tsRNA-5001a was predominantly localized in the cytoplasm (Figure 5).

\section{$R N A$ sequencing}

\section{Analysis of differentially expressed genes after knockdown}

After extracting total RNA from the knockdown group and control group samples of PC9 cells (3 biological replicates for each), we removed ribosomal RNA, built a library of the remaining RNA, and performed high-throughput transcriptome sequencing. RNA-seq data of this study are available in the Gene Expression Omnibus (GEO) database (GEO accession number: GSE179341, https://www.ncbi. nlm.nih.gov/geo/query/acc.cgi?acc=GSE179341). After the raw data were quality controlled with Fastqc software, the HISAT2 software was used to compare the data to the human reference genome and to perform gene annotation. Subsequently, we used DESeq2 for differential expression analysis. The differentially expressed gene screening 

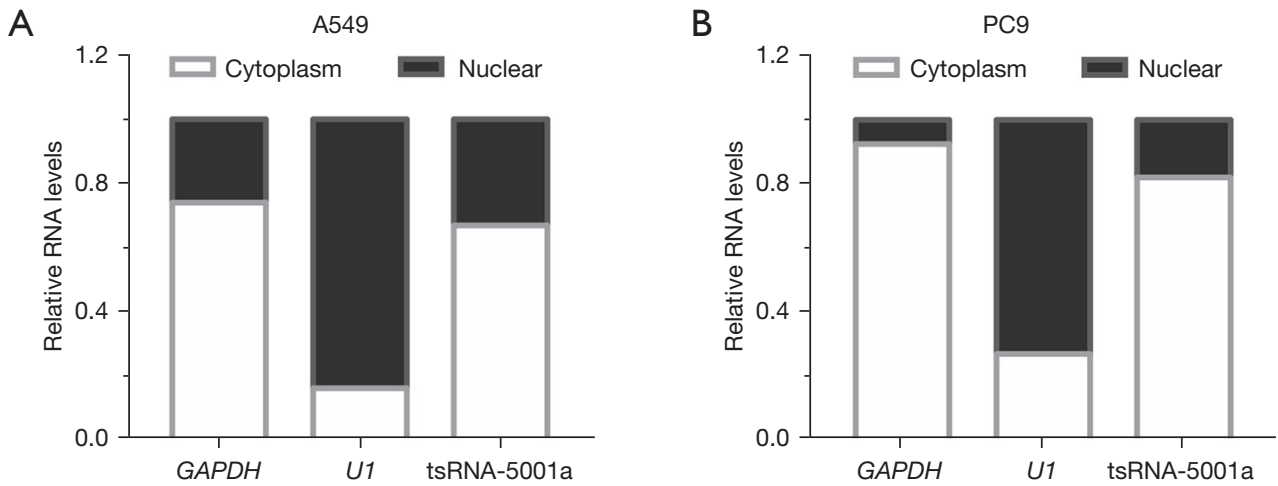

Figure 5 Cell localization of tsRNA-5001a was performed using a nucleo-cytoplasmic assay. After the separation of the cytoplasm and nucleoplasm, RNA was extracted from all fractions, and tsRNA-5001a expression was measured by RT-qPCR. In A549 cells, the expression ratio of tsRNA-5001a was $67.1 \%$ in the cytoplasm and 32.9\% in the nucleus (A). In PC9 cells, the expression ratio of tsRNA5001a was $82.1 \%$ in the cytoplasm and $17.9 \%$ in the nucleus (B). RT-qPCR, real-time quantitative polymerase chain reaction; GAPDH, glyceraldehyde-3-phosphate dehydrogenase; U1, U1 small nuclear 1.

conditions were set to an adjusted $\mathrm{P}$ value $<0.05$, absolute value of $\log 2$ fold change $>1$, and base mean $>5$. Under these conditions, we obtained a total of 213 significant differentially expressed genes, of which 3 genes were downregulated and 210 genes were upregulated in the knockdown group. The results of differentially expressed genes are displayed on the heat map (Figure 6A). The expression levels of this group of genes were relatively consistent within the group, but the expression levels between the groups were significantly different. The selected differentially expressed gene set only accounts for a small part of the total genes (Figure 6B), but the original expression count (read count) of this group of genes could be used to logically divide the sample into two groups (Figure 6C). The results suggested that the differentially expressed genes we screened may be the effect gene set of antagomir treatment.

Subsequently, we performed a functional enrichment analysis of the genes with increased expression. After using the ClusterProfiler software package (RStudio, Boston, MA, USA) to perform a biological progress-based enrichment analysis on the gene list, the results showed that these upregulated genes were significantly related to 10 pathways, including epithelial cell proliferation, regulation of epithelial cell proliferation, and positive regulation of ion transport, among others (see GO bubble plot for specific names; Figure $6 D$ ). We then used the gProfiler database to analyze the enrichment of differentially upregulated genes in the KEGG signaling pathway. The results showed that there were three signaling pathways, including melanoma, transcriptional misregulation in cancer and the MAPK signaling pathway, which involved 16 genes. The detailed results are shown in the Circos diagram (Figure 6E). The results of the enrichment analysis suggested that after the cell lines were treated with tsRNA-5001a antagomir, the increased gene expression was closely related to some important signal pathways, such as cell proliferation and tumor transcription regulation.

\section{Analysis of the interaction between tsRNA-5001a antagomir and genes}

Knocking tsRNA-5001a down with antagomir led to significant changes in gene expression in the transcriptional misregulation in the cancer signaling pathway involved in cancer progression, with 9 genes mainly contributing to the changes. These genes interact with one another to form a tight-knit network of interactions with a few intermediate proteins in the signaling pathways that drive the abnormal regulation of tumor transcription (Figure $7 A$ ). We speculate that tsRNA-5001a may have a direct association with the target messenger RNA (mRNA) that causes a decrease in mRNA expression (this association may be similar to the effect of microRNA binding to mRNA causing a decrease in mRNA stability and expression) and that knockdown of tsRNA-5001a can cause reverse changes in the binding of mRNA. Therefore, we compared the tsRNA-5001a sequence with the promoter sequence of mRNA in the transcriptional misregulation in cancer signaling pathway using the miMap database. The results showed that tsRNA5001a might target the TRAF1, NR4A3, and GADD45G genes (Figure 7B). These results suggest that tsRNA-5001a 
A

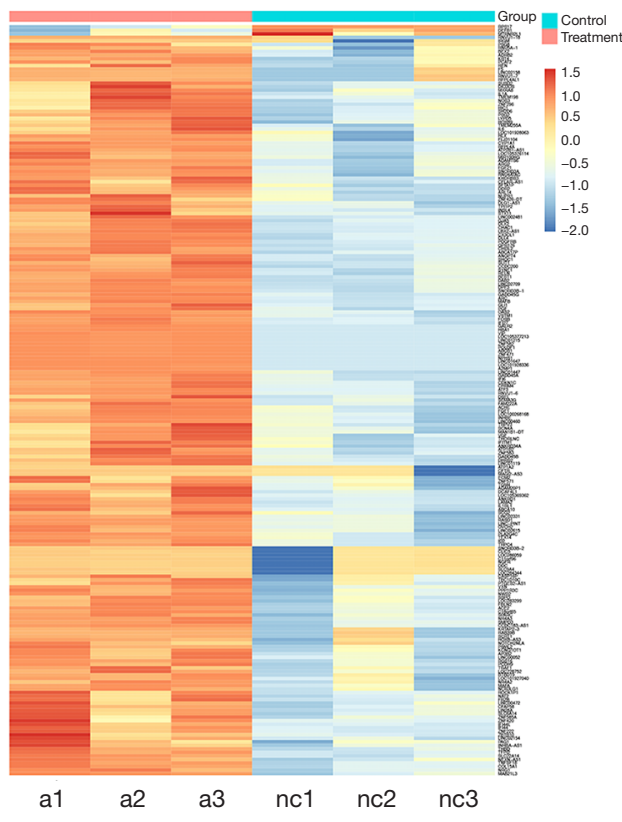

$\mathrm{D}$

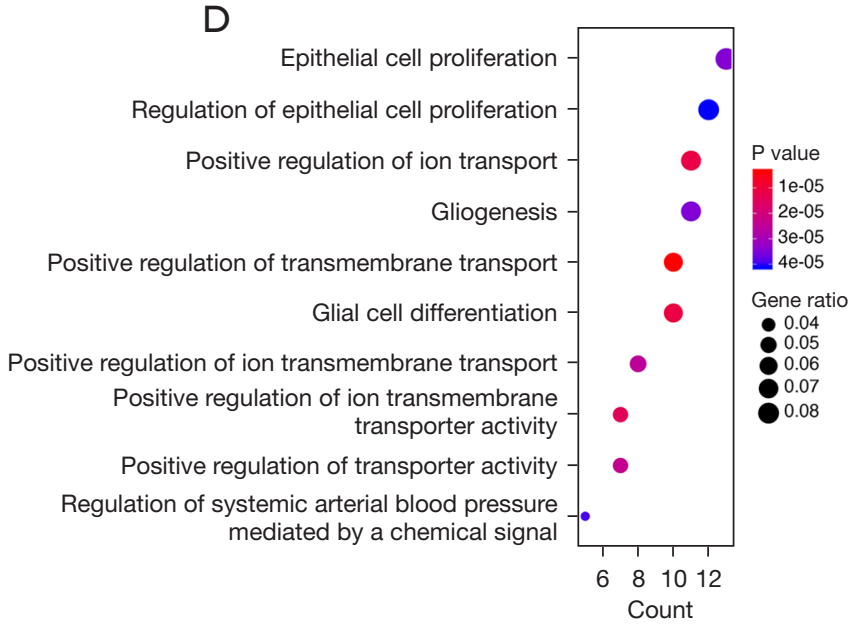

B
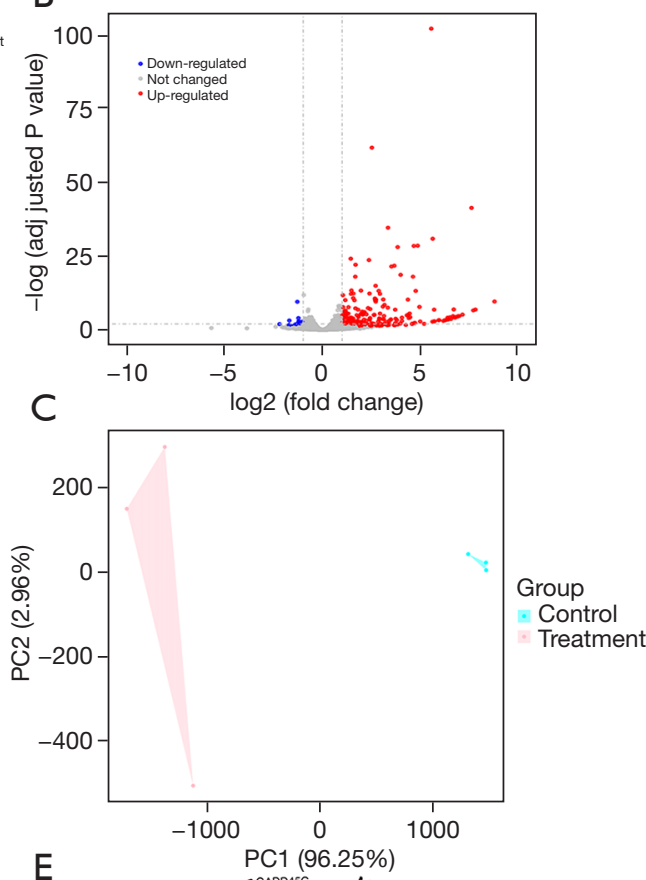

E

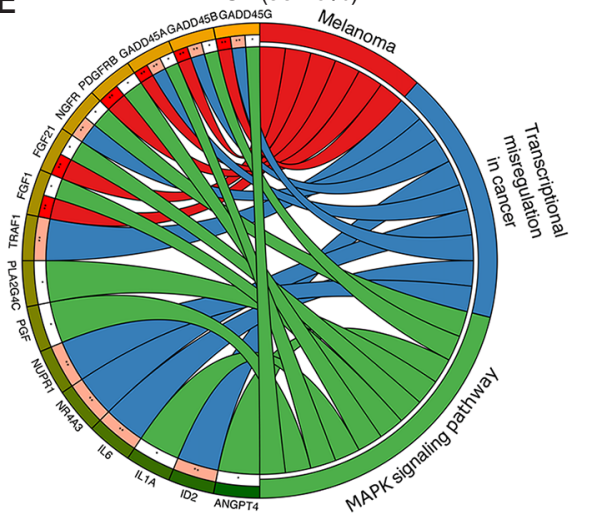

Figure 6 Analysis of differentially expressed genes after knockdown. Results of differentially expressed genes are shown on the heat map (A). A total of 213 differentially expressed genes were obtained, among which 3 showed decreased expression in the knockout group and 210 showed increased expression in the knockout group (B). The sample was logically divided into two groups according to the read count of the gene expression in this group (C). Bubble plot of the GO enrichment result (D). Functional enrichment analysis of the genes with increased expression showed that the upregulated genes were significantly correlated with 10 pathways. The Y-axis corresponds to the enriched pathway, and the $\mathrm{X}$-axis indicates the number of differential genes in that pathway. The bubble color represents the $\mathrm{P}$ value of the GO term, while the bubble size represents the ratio of differential genes in the pathway to all genes in the pathway. The Gprofiler database was used to analyze the enrichment of differentially upregulated genes in the KEGG signaling pathway (E). GO, Gene Ontology; KEGG, Kyoto Encyclopedia of Genes and Genomes; MAPK signaling pathway, mitogen-activated protein kinase signaling pathway; GADD45G, growth arrest and DNA damage inducible gamma; $G A D D 45 B$, growth arrest and DNA damage inducible beta; $G A D D 45 A$, growth arrest and DNA damage inducible alpha; $P D G F R B$, platelet derived growth factor receptor beta; $N G F R$, nerve growth factor receptor; $F G F 21$, fibroblast growth factor 21; FGF1, fibroblast growth factor 1; TRAF1, TNF receptor associated factor 1; PLA2G4C, phospholipase A2 group IVC; $D G F$, placental growth factor; NUPR1, nuclear protein 1, transcriptional regulator; $N R 4 A 3$, nuclear receptor subfamily 4 group A member 3 ; IL6, interleukin 6; IL1A, interleukin 1 alpha; ID2, inhibitor of DNA binding 2; ANGPT4, angiopoietin 4. 
A

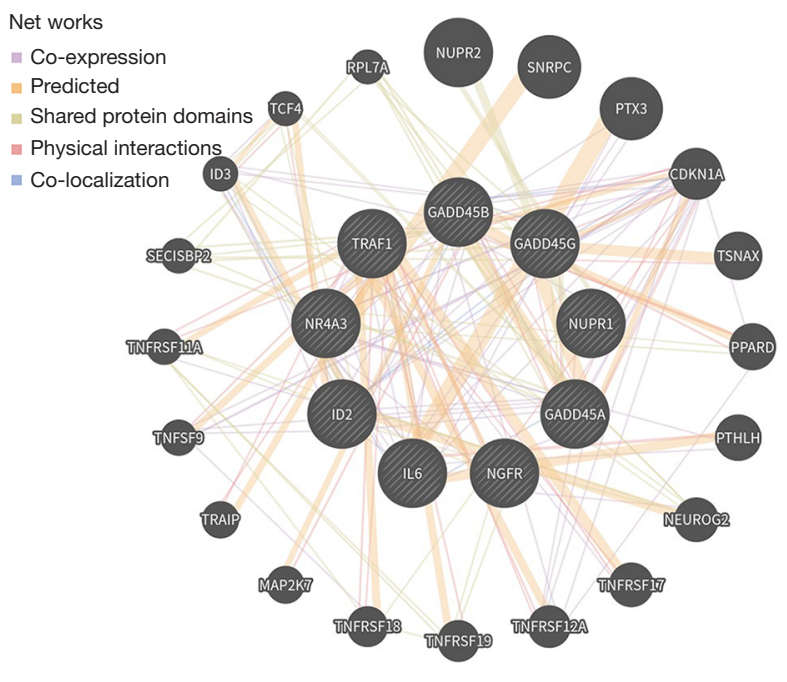

C

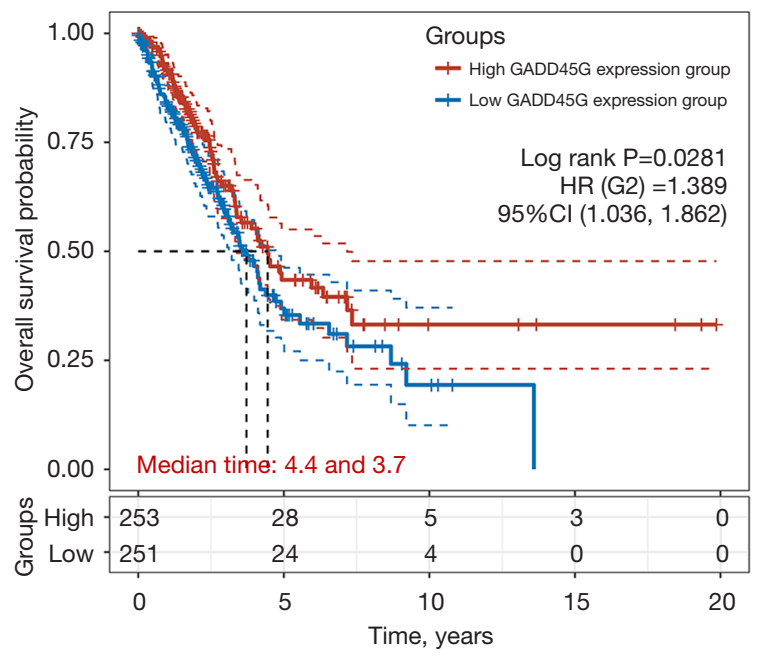

B
TRAF1-target site 1

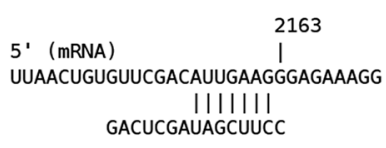

TRAF1-target site 2

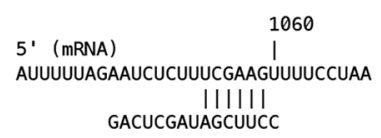

NR4A3 target site

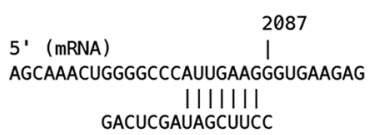

GADD45G target site

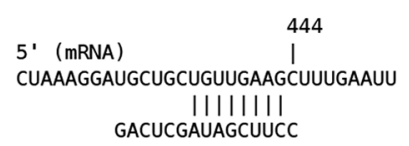
GACUCGAUAGCUUCC
D

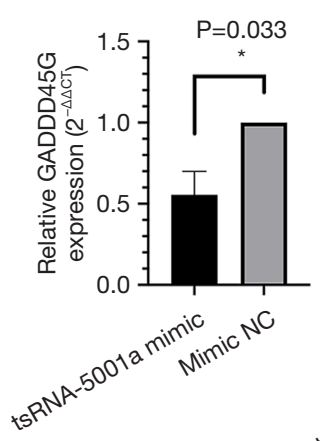

PC9

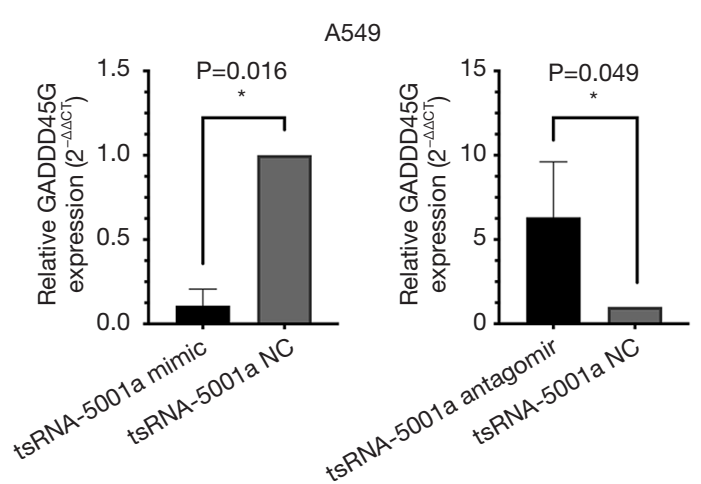

Figure 7 Analysis of the interaction between tsRNA-5001a antagomir and genes. A diagram of the interrelationship network composed of key genes in the transcriptional misregulation in cancer pathway (A). The sequence of tsRNA-5001a was compared with that of mRNA with the miMap database. The results showed that tsRNA-5001a may bind to TRAF1, NR4A3, and GADD45G genes (B). Survival analysis was performed on raw counts of RNA sequencing data and corresponding clinical information in lung adenocarcinoma patients obtained from The Cancer Genome Atlas (TCGA) database (C). The expression of GADD45G in overexpressed and knockdown PC9 cells and A549 cells was further verified by RT-qPCR (D). *, $\mathrm{P}<0.05$; **, $\mathrm{P}<0.01$. TRAF1, TNF receptor associated factor $1 ; N R 4 A 3$, nuclear receptor subfamily 4 group A member 3; GADD45G, growth arrest and DNA damage inducible gamma; RT-qPCR, real-time quantitative polymerase chain reaction; NC, negative control.

has the potential to bind to tumor regulatory genes and inhibit the expression level of tsRNA-5001a, which would in turn enhance the expression level of target genes.
For the functional study of target genes, we screened 513 patients with lung adenocarcinoma from the TCGA database, which included transcriptome sequencing, 
mutation information, and clinical information. We then performed a survival analysis based on the expression of TRAF1, NR4A3, and GADD45G (the median value of gene expression was used as the cutoff value, and high expression and low expression groups were assigned) in 504 lung adenocarcinoma patients ( 9 patients out of 513 were not included in the survival analysis because of the lack of survival time data). Through survival analysis, we found that the prognosis of patients with low GADD45G expression was significantly worse than those with high $G A D D 45 G$ expression, suggesting that $G A D D 45 G$ may have the function of inhibiting tumor development and improving prognosis (Figure 7C). Therefore, we speculate that the high expression of tsRNA-5001a accelerates the proliferation of lung adenocarcinoma cells and worsens the prognosis of patients with lung adenocarcinoma. This phenomenon is caused by the combination of tsRNA-5001a and $G A D D 45 G$, which reduces the stability of $G A D D 45 G$ and leads to the downregulation of GADD45G expression, thus inhibiting the antitumor function of $G A D D 45 G$. In addition, we found a significant decrease in $G A D D 45 G$ expression in patients with EGFR mutations in TCGA database, suggesting that $G A D D 45 G$ may be a characteristic gene of EGFR mutations; however, this relationship needs to be further explored (Figure S3).

To further validate the target gene results obtained from the RNA-seq screening, we assessed the changes of the target gene in the PC9 cells and A549 cells and found that $G A D D 45 G$ was downregulated in the overexpression group and significantly upregulated in the knockdown group. These results were consistent with those of RNA-seq, eliminating the possibility of false positives in RNA-seq and thus further confirming that $G A D D 45 G$ might be the target gene for tsRNA-5001a (Figure 7D).

\section{Discussion}

tsRNAs are one of the oldest small RNAs presented in all domains of life (Archaea, Bacteria, and Eukarya), and they are produced by the cleavage of tRNAs (14). By virtue of its conserved sequence, rich RNA modification, and proteinbinding ability, emerging recent studies have confirmed tsRNA's multiple roles in basic biological processes, such as gene silencing, reverse transcription transposition, ribosomal biogenesis, cell differentiation, and others (14-17).

In this study, we analyzed the microarray data and found that tsRNA-5001a was significantly upregulated in lung adenocarcinoma. After that, we preliminarily selected
tsRNA-5001a with obvious function after screening by cell proliferation assay, so we chose tsRNA-5001a for the study. Then, RT-qPCR was used to detect the expression of tsRNA-5001a in postoperative samples of patients with lung adenocarcinoma. It was found that the expression of tsRNA5001a was negatively correlated with DFS, suggesting that high expression of tsRNA-5001a can increase the risk of postoperative recurrence and affect the prognosis of patients with primary lung adenocarcinoma. Therefore, we speculate that tsRNA-5001a is a potential indicator that can predict DFS in postoperative patients with lung adenocarcinoma. After overexpression and knockdown of tsRNA-5001a in lung adenocarcinoma cell lines, we found through CCK8 cell proliferation assay and clone formation experiments that overexpression of tsRNA-5001a could significantly accelerate the proliferation of lung adenocarcinoma cells. Conversely, knockdown of tsRNA-5001a could significantly inhibit the proliferation of lung adenocarcinoma cells. Additionally, the subcellular localization of tsRNA-5001a was determined by nucleoplasmic separation experiments. The results showed that tsRNA-5001a was mainly expressed in cytoplasm. Subsequently, we used RNA-seq to screen the downstream gene and pathway changes in the knockdown group and knockdown control group of PC9 cells. The results showed that the pathways involved in cell proliferation and tumor transcription regulation were significantly changed after tsRNA-5001a knockdown. We further identified GADD45G as the target gene of tsRNA-5001a through miMap binding site prediction and TCGA database. According to the findings of this study and previous studies, we speculate that tsRNA-5001a can bind with $G A D D 45 G$, which is an antitumor gene, to reduce its stability, inhibit its antitumor function, and further accelerate tumor proliferation, thus worsening the prognosis of lung adenocarcinoma. The findings of this study may help inform further investigation into the pathogenesis of lung adenocarcinoma and aid in identifying novel therapeutic targets.

Previous studies have found that tsRNAs can exert their biological functions by regulating protein translation initiation, argonaute (AGO)-dependent translation inhibition, AGO-independent translation regulation, transposon regulation, and other processes $(15,18-25)$. Currently, the mechanisms of tsRNA reported by existing research involve six principal areas.

(I) Regulation of protein translation initiation: Studies found that $5^{\prime}$ tsRNA ${ }^{\mathrm{Ala}}$ and $5^{\prime}$ tsRNA ${ }^{\mathrm{Cys}}$ with terminal oligo-G motifs (TOG) can form 
intermolecular RNA G-quadruplexes (RG4) to replace eukaryotic translation initiation factor 4G/eukaryotic translation initiation factor $4 \mathrm{E}$ (eIF4G/eIF4E) on the structure of the mRNA cap (m7GTP), thus inhibiting translation (21);

(II) AGO-dependent translation inhibition: tsRNA can inhibit the translation of mRNA in a sequencespecific way by binding with AGO protein (AGO protein is the key protein of RNA-mediated posttranscriptional gene regulation, which provides an anchor point for small ncRNAs to degrade target genes or inhibit translation), suggesting that tsRNA can regulate the gene expression, such as microRNA to some extent $(18,23)$;

(III) Translation regulation-independent of AGO: A recent study have found that a 3' tsRNA with a length of $22 \mathrm{nt}$ does not bind to any known AGO protein, but specifically binds to the mRNA of ribosomal proteins RPS28 and RPS15 through base pairing, which unfolds the duplex secondary structure of the target sites in mRNA and increases the translation of ribosomal proteins (15). In addition, some studies have found that some $3^{\prime}$ tsRNAs and $5^{\prime}$ tsRNAs can bind to small ribosomal subunits and aminoacyl tRNA synthetases to inhibit translation by interfering with the aminoacylation of Trna (24);

(IV) tsRNA can competitively bind with YBX1 (Y-box binding protein 1 ), which makes the transcripts of many oncogenes bound with YBX1 lose their stability and inhibit tumor metastasis $(19,25)$;

(V) tsRNA can form multi-synthetase complex (MSC) and affect translation (22);

(VI) tsRNA is involved in retrotransposon regulation (20). tRNA is the most abundantly modified RNA in the body (26). Studies have confirmed that the modification of tRNA can affect the cleavage of tRNA by endonuclease and regulate the production of tsRNA (27). Since tsRNA is derived from tRNA, tsRNA also contains abundant modifications. Studies have shown that rich modifications of tsRNA can change its secondary structure and stability, as well as affect other biological functions of tsRNA $(28,29)$. As the rich modification of tsRNA affects the subsequent reverse transcription and PCR, after the total RNA was extracted from the tissue sample examined in this study, we completed a pretreatment step to remove the rich modification of tsRNA.

tsRNA has attracted the attention of oncology researchers from all over the world. A number of studies have shown that tsRNA is abnormally expressed in a variety of cancers. The expression of tsRNA is also altered in individuals in whom cancer has progressed. Studies have shown that tsRNA plays a key role in human cancer by participating in a variety of biological functions, including gene expression and silencing, epigenetic regulation and translation regulation (18,30,31). A study conducted by Lee and colleagues found that the expression of $3^{\prime}$ tsRNA ${ }^{\text {Ser (TGA) }}$ was positively associated with the proliferation of prostate cancer cell lines and promoted the transformation of cancer cells from G2 to $M$ stages (32). In the study of renal clear cell carcinoma, Nientiedt $e t$ al. found that the expression level of 5'tsRNA ${ }^{\mathrm{Val}(\mathrm{AAC})}$ was significantly related to the stage and grade of the tumor (33). In addition, in a study of nonsmall cell lung cancer, it was found that $5^{\prime}$ tsRNA ${ }^{\mathrm{LEU}(\mathrm{CAG})}$ was highly expressed in tumor tissues, and inhibition of 5 tsRNA ${ }^{\mathrm{LEU}(\mathrm{CAG})}$ could significantly inhibit cell proliferation (34). Furthermore, a previous study using RNA deep sequencing and bioinformatics analysis showed that serum 5 '-tsRNA is a potential biomarker for breast cancer (35).

We analyzed the data of RNA-seq and TCGA database and speculated that GADD45G may be the target gene of tsRNA-5001a. GADD45G is an important member of the Growth Arrest and DNA damage-inducible 45 (GADD45) gene family. This gene family is a family of stress response genes that negatively regulate cell proliferation. GADD45G can participate in DNA damage repair, play an important role in apoptosis, cell survival and signal transduction, and is also closely related to tumor occurrence, development and prognosis (36). Previous studies have shown that GADD45G is aberrantly down-regulated in a variety of tumors, such as lung cancer, esophageal cancer, pancreatic cancer, cervical cancer, and so on. It has been demonstrated that down-regulation of GADD45G is associated with tumor progression, metastasis and poor prognosis (36-38). Since this study found that tsRNA-5001a was associated with an increased risk of postoperative recurrence in patients with lung adenocarcinoma, and it was speculated that tsRNA-5001a may act through GADD45G. So, what is the role of some DNA repair genes with similar functions as GADD45G in lung adenocarcinoma patients undergoing postoperative treatment? Martin Pesta and his colleagues conducted a research in this area and found that among patients undergoing surgery, those with low expression of DNA repair genes had a poor prognosis (39).

In this study, tsRNA-5001a was found to be abnormally expressed in lung adenocarcinoma tissues and correlated 
with prognosis of lung adenocarcinoma patients. We can thus speculate that tsRNA-5001a can serve as a biomarker for the diagnosis and treatment of lung adenocarcinoma in the future.

\section{Conclusions}

tsRNA-5001a was found to accelerate the proliferation of lung adenocarcinoma cells, and its high expression may increase the risk of postoperative recurrence of lung adenocarcinoma patients. RNA-seq and TCGA database analysis revealed that $G A D D 45 G$ may be the target gene of tsRNA-5001a. Targeting tsRNA-5001a may represent a novel approach in treating lung cancer.

\section{Acknowledgments}

The authors are grateful to all patients included in this study and are especially grateful to Chengtao Yu for his contribution to this study. The authors also appreciate the academic support from AME Lung Cancer Collaborative Group.

Funding: This study was supported by National Key Research and Development Program of China (No. 2018YFC1313604) and the Chinese Society of Clinical Oncology (CSCO; No. Y-2019AZZD-0038). The funders played no role in the design or conduct of this research.

\section{Footnote}

Reporting Checklist: The authors have completed the MDAR reporting checklist. Available at https://dx.doi. org/10.21037/tlcr-21-829

Data Sharing Statement: Available at https://dx.doi. org/10.21037/tlcr-21-829

Conflicts of Interest: All authors have completed the ICMJE uniform disclosure form (available at https://dx.doi. org/10.21037/tlcr-21-829). All authors reported that this study was supported by National Key Research and Development Program of China (No. 2018YFC1313604) and the Chinese Society of Clinical Oncology (CSCO; No. Y-2019AZZD-0038). CBS serves as an unpaid editorial board member of Translational Lung Cancer Research. The authors have no other conflicts of interest to declare.

Ethical Statement: The authors are accountable for all aspects of the work in ensuring that questions related to the accuracy or integrity of any part of the work are appropriately investigated and resolved. This research was approved by the Ethics Committee of Shanghai Chest Hospital, Shanghai Jiao Tong University (No. KS(Y)21105). This study complied with the Declaration of Helsinki (as revised in 2013) and obtained the informed consent of all patients.

Open Access Statement: This is an Open Access article distributed in accordance with the Creative Commons Attribution-NonCommercial-NoDerivs 4.0 International License (CC BY-NC-ND 4.0), which permits the noncommercial replication and distribution of the article with the strict proviso that no changes or edits are made and the original work is properly cited (including links to both the formal publication through the relevant DOI and the license). See: https://creativecommons.org/licenses/by-nc-nd/4.0/.

\section{References}

1. Sung H, Ferlay J, Siegel RL, et al. Global Cancer Statistics 2020: GLOBOCAN Estimates of Incidence and Mortality Worldwide for 36 Cancers in 185 Countries. CA Cancer J Clin 2021;71:209-49.

2. Siegel RL, Miller KD, Fuchs HE, et al. Cancer Statistics, 2021. CA Cancer J Clin 2021;71:7-33.

3. Yuan C, Chen H, Tu S, et al. A systematic dissection of the epigenomic heterogeneity of lung adenocarcinoma reveals two different subclasses with distinct prognosis and core regulatory networks. Genome Biol 2021;22:156.

4. Ling H, Girnita L, Buda O, et al. Non-coding RNAs: the cancer genome dark matter that matters! Clin Chem Lab Med 2017;55:705-14.

5. Zhang X, Xie K, Zhou H, et al. Role of non-coding RNAs and RNA modifiers in cancer therapy resistance. Mol Cancer 2020;19:47.

6. Park J, Ahn SH, Shin MG, et al. tRNA-Derived Small RNAs: Novel Epigenetic Regulators. Cancers (Basel) 2020;12:2773.

7. Jia Y, Tan W, Zhou Y. Transfer RNA-derived small RNAs: potential applications as novel biomarkers for disease diagnosis and prognosis. Ann Transl Med 2020;8:1092.

8. Zong T, Yang Y, Zhao H, et al. tsRNAs: Novel small molecules from cell function and regulatory mechanism to therapeutic targets. Cell Prolif 2021;54:e12977.

9. Xue M, Shi M, Xie J, et al. Serum tRNA-derived small RNAs as potential novel diagnostic biomarkers for 
pancreatic ductal adenocarcinoma. Am J Cancer Res 2021;11:837-48.

10. Tao EW, Wang HL, Cheng WY, et al. A specific tRNA half, 5'tiRNA-His-GTG, responds to hypoxia via the HIF $1 \alpha / A N G$ axis and promotes colorectal cancer progression by regulating LATS2. J Exp Clin Cancer Res 2021;40:67.

11. Liu W, Liu Y, Pan Z, et al. Systematic Analysis of tRNADerived Small RNAs Discloses New Therapeutic Targets of Caloric Restriction in Myocardial Ischemic Rats. Front Cell Dev Biol 2020;8:568116.

12. Farina NH, Scalia S, Adams CE, et al. Identification of tRNA-derived small RNA (tsRNA) responsive to the tumor suppressor, RUNX1, in breast cancer. J Cell Physiol 2020;235:5318-27.

13. Zhang J, Li L, Luo L, et al. Screening and potential role of tRFs and tiRNAs derived from tRNAs in the carcinogenesis and development of lung adenocarcinoma. Oncol Lett 2021;22:506.

14. Chen Q, Zhang X, Shi J, et al. Origins and evolving functionalities of tRNA-derived small RNAs. Trends Biochem Sci 2021;46:790-804.

15. Kim HK, Fuchs G, Wang S, et al. A transfer-RNAderived small RNA regulates ribosome biogenesis. Nature 2017;552:57-62.

16. Wang T, Mei J, Li X, et al. A novel tsRNA-16902 regulating the adipogenic differentiation of human bone marrow mesenchymal stem cells. Stem Cell Res Ther 2020;11:365.

17. Domingues S, Moreira RN, Andrade JM, et al. The role of RNase $\mathrm{R}$ in trans-translation and ribosomal quality control. Biochimie 2015;114:113-8.

18. Kumar P, Anaya J, Mudunuri SB, et al. Meta-analysis of tRNA derived RNA fragments reveals that they are evolutionarily conserved and associate with AGO proteins to recognize specific RNA targets. BMC Biol 2014;12:78.

19. Lyons SM, Achorn C, Kedersha NL, et al. YB-1 regulates tiRNA-induced Stress Granule formation but not translational repression. Nucleic Acids Res 2016;44:6949-60.

20. Zhang Y, Shi J, Chen Q. tsRNAs: new players in mammalian retrotransposon control. Cell Res 2017;27:1307-8.

21. Lyons SM, Gudanis D, Coyne SM, et al. Identification of functional tetramolecular RNA G-quadruplexes derived from transfer RNAs. Nat Commun 2017;8:1127.

22. Keam SP, Sobala A, Ten Have S, et al. tRNA-Derived RNA Fragments Associate with Human Multisynthetase
Complex (MSC) and Modulate Ribosomal Protein Translation. J Proteome Res 2017;16:413-20.

23. Maute RL, Schneider C, Sumazin P, et al. tRNA-derived microRNA modulates proliferation and the DNA damage response and is down-regulated in B cell lymphoma. Proc Natl Acad Sci U S A 2013;110:1404-9.

24. Mleczko AM, Celichowski P, Bąkowska-Żywicka K. Transfer RNA-derived fragments target and regulate ribosome-associated aminoacyl-transfer RNA synthetases. Biochim Biophys Acta Gene Regul Mech 2018. [Epub ahead of print].

25. Goodarzi H, Liu X, Nguyen HC, et al. Endogenous tRNADerived Fragments Suppress Breast Cancer Progression via YBX1 Displacement. Cell 2015;161:790-802.

26. Pan T. Modifications and functional genomics of human transfer RNA. Cell Res 2018;28:395-404.

27. Lyons SM, Fay MM, Ivanov P. The role of RNA modifications in the regulation of tRNA cleavage. FEBS Lett 2018;592:2828-44.

28. Zhang Y, Zhang Y, Shi J, et al. Identification and characterization of an ancient class of small RNAs enriched in serum associating with active infection. J Mol Cell Biol 2014;6:172-4

29. Guzzi N, Cieśla M, Ngoc PCT, et al. Pseudouridylation of tRNA-Derived Fragments Steers Translational Control in Stem Cells. Cell 2018;173:1204-1216.e26.

30. Yu M, Lu B, Zhang J, et al. tRNA-derived RNA fragments in cancer: current status and future perspectives. J Hematol Oncol 2020;13:121.

31. Schorn AJ, Gutbrod MJ, LeBlanc C, et al. LTRRetrotransposon Control by tRNA-Derived Small RNAs. Cell 2017;170:61-71.e11.

32. Lee YS, Shibata Y, Malhotra A, et al. A novel class of small RNAs: tRNA-derived RNA fragments (tRFs). Genes Dev 2009;23:2639-49.

33. Nientiedt $M$, Deng M, Schmidt D, et al. Identification of aberrant tRNA-halves expression patterns in clear cell renal cell carcinoma. Sci Rep 2016;6:37158.

34. Shao Y, Sun Q, Liu X, et al. tRF-Leu-CAG promotes cell proliferation and cell cycle in non-small cell lung cancer. Chem Biol Drug Des 2017;90:730-8.

35. Dhahbi JM, Spindler SR, Atamna H, et al. Deep Sequencing of Serum Small RNAs Identifies Patterns of 5' tRNA Half and YRNA Fragment Expression Associated with Breast Cancer. Biomark Cancer 2014;6:37-47.

36. Wan Mohd Tajuddin WNB, Abas F, Othman I, et al. Molecular Mechanisms of Antiproliferative and Apoptosis Activity by 1,5-Bis(4-Hydroxy-3-Methoxyphenyl)1,4- 
Pentadiene-3-one (MS13) on Human Non-Small Cell Lung Cancer Cells. Int J Mol Sci 2021;22:7424.

37. Guo W, Zhu T, Dong Z, et al. Decreased expression and aberrant methylation of Gadd45G is associated with tumor progression and poor prognosis in esophageal squamous cell carcinoma. Clin Exp Metastasis 2013;30:977-92.

38. Ying J, Srivastava G, Hsieh WS, et al. The stressresponsive gene GADD45G is a functional tumor

Cite this article as: $\mathrm{Hu}$ F, Niu Y, Mao X, Cui J, Wu X, Simone CB 2nd, Kang HS, Qin W, Jiang L. tsRNA-5001a promotes proliferation of lung adenocarcinoma cells and is associated with postoperative recurrence in lung adenocarcinoma patients. Transl Lung Cancer Res 2021;10(10):3957-3972. doi: 10.21037/ tlcr-21-829 suppressor, with its response to environmental stresses frequently disrupted epigenetically in multiple tumors. Clin Cancer Res 2005;11:6442-9.

39. Pesta M, Kulda V, Fiala O, et al. Prognostic significance of ERCC1, RRM1 and BRCA1 in surgically-treated patients with non-small cell lung cancer. Anticancer Res 2012;32:5003-10. 


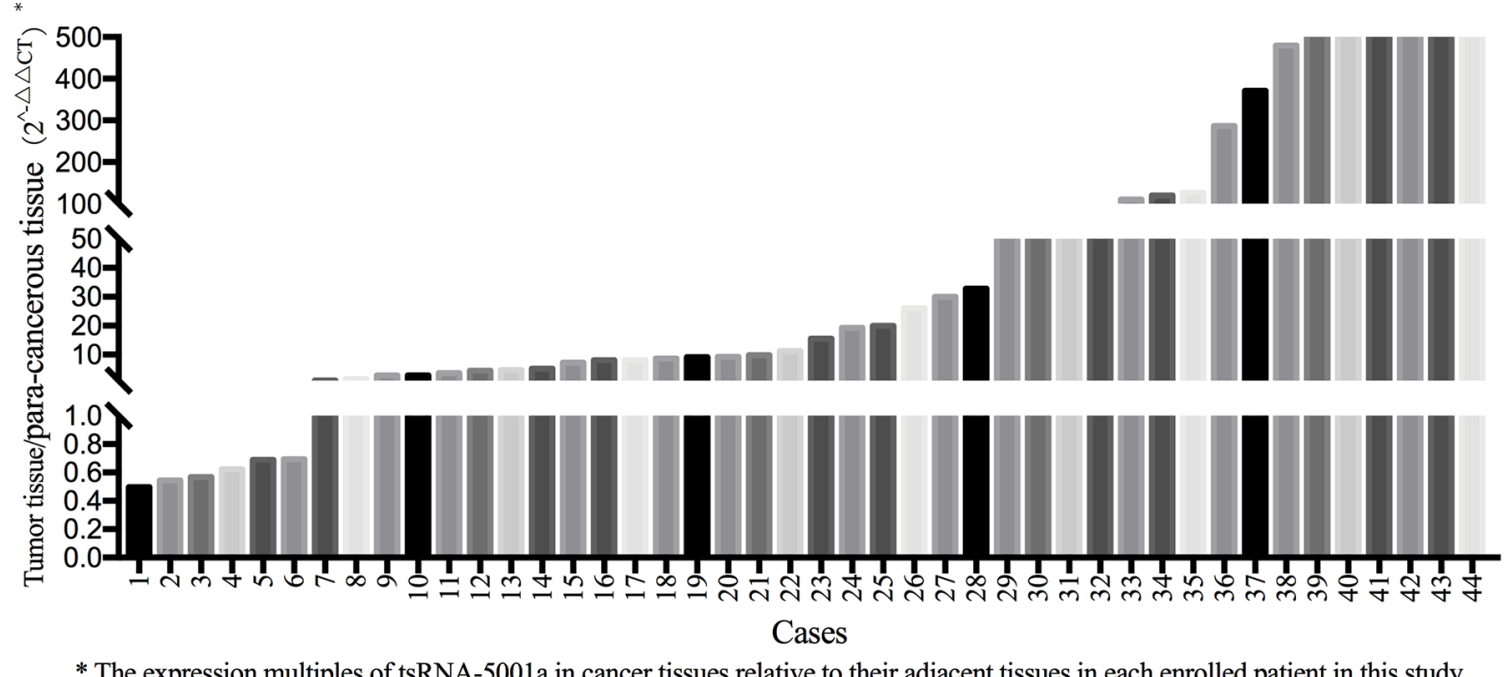

Figure S1 The expression level of tsRNA-5001a in tumor and paired adjacent tissues of enrolled patients was detected by RT-qPCR. The abscissa represents the patients, and the ordinate represents the expression level of tsRNA-5001a in the tumor tissues relative to the paired adjacent tissues. The $2^{-\Delta \mathrm{CT}}$ method was used to calculate the relative expression level. RT-qPCR, real-time quantitative polymerase chain reaction.

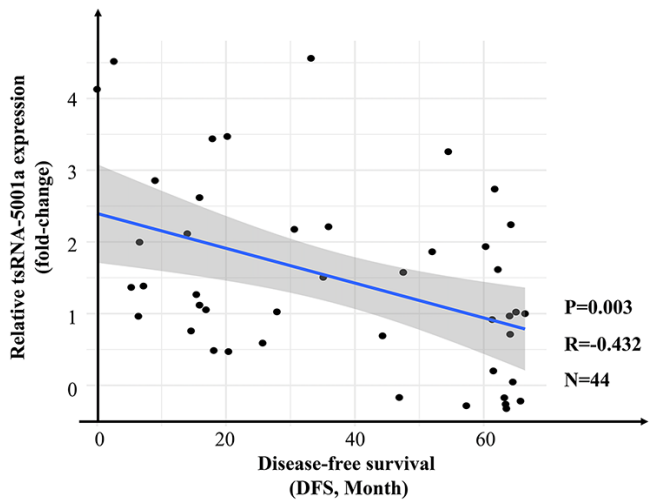

Figure S2 Correlation between tsRNA-5001a relative expression level and DFS. DFS, disease-free survival.

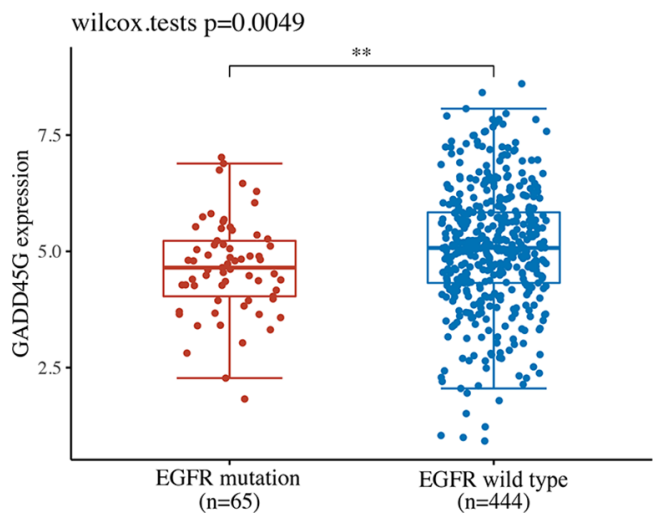

Figure $\mathbf{S} 3$ GADD45G expression in EGFR-mutant and wildtype patients (4 patients out of 513 patients were not included due to EGFR mutation information being not available). ${ }^{* *}, \mathrm{P}<0.01$. $G A D D 45 G$, growth arrest and DNA damage inducible gamma; $E G F R$, epidermal growth factor receptor. 